\title{
A Dynamic Time-slot Assignment for Ground Wireless Sensor Network
}

\author{
Gao Ren, Tang Long and Hu Wen \\ The School of Information Engineering, Hubei University of Economics, Wuhan, \\ China \\ Gaoren@whu.edu.cn,huwen@whu.edu.cn
}

\begin{abstract}
This paper is on the background of the applied communications of automated vehicle in the multi-hop environment. The paper provides a dynamic time-slot assignment protocol for the ground wireless sensor network (WSN), based on TDMA. By interacting of the two-hop neighboring nodes information, the protocol finishes the dynamic time-slot assignment, realizes the efficient and fair sharing of channel, reduces the transfer delay of the end-to-end burst data, meets the communication demand of assignment on-demand in WSN, and prolongs the life-time of the network with the virtual backbone mechanism. The theoretical analysis and simulation results showed that the protocol provided has better adaptability for the burst network services and topological variation, comparing to the traditional static mac layer protocol in the TDMA system.
\end{abstract}

Keywords: wireless sensor network (WSN), mac layer protocol (MAC), time division multiple address (TDMA), automated vehicle

\section{Background}

WSN has widely used in various fields, especially in the military field. It is well suited to use in the wretched battlefield environment, including monitoring our forces, materials and equipment, keeping watch over the conflict zones, detecting the topography and defensive of the enemy, aiming the targets, assessing the damage, detecting the nuclear, biological and chemical attacks. WSN on the ground is disposed into three parts: the lowest part is the smallest and lowest power consumption attended sensor, the second part is the directional sensor which could assess the azimuth and the third part is the confirmed sensor which has a imaging ability. The first two parts only need slow communications (kbps) between each other and the communication distance is 100-400 meters. The confirmed sensor in the third part need two-speed or two transceivers (Mbps), the one is responsible for the communication with the lower node and the other one is responsible for the communication with the directorial node in a distance which could not exceed $10 \mathrm{~km}$.

Based on the communication of the ground sensor by using unmanned vehicle in mobile multi-hop, a MAC protocols for the ground wireless network is designed, a TDMA-based dynamic TSA protocol is proposed, this protocol can complete the TSA process by the communication between two neighboring nodes, which can realize the sharing of the high-quality and fair-minded signal path, fulfilling the demand to distribute the communication according to need for the ground wireless network at the same time.

This review is followed by an introduction. A brief summary of some of the relevant concepts is presented in Section 2.Section 3 demonstrates the protocol design. Section 4 is the theoretical performance study of the protocol. In Section 5, we discuss the preliminary results of a simulation study. Finally, conclusions and future work are summarized. 


\section{Related work}

\subsection{Correlation Study}

There are such three types of time-slot assignment in MAC protocol, including static, dynamic and a mixed assignment. And the dynamic one is focused on in this paper. It has a great advantage in a wireless sensor network, for example, flexible assignment of timeslot dynamic topology support and so on.

There exist such MAC protocols based on TDMA as TRAMA [1], LMAC and RTLink, in which nodes are capable of communication using proprietary TS. TRAMA supports node communication and network management through planning and CSMAbased TS, and LMAC supports TSA in lightweight load condition and a neighbor negotiation TS reservation assignment between two biggest hops, while RT-Link supports a static TSA after global clock synchronization. Centralized static TSA is the common feature of above three protocols which cannot meet the need of burst traffic.

The CSMA based protocol suffers from unavoidable collisions when more than one vehicle decreases their back off counter to zero and access channel simultaneously, and it may cause unbounded access delay and serious packet loss especially in high vehicle density scenario [4]. It introduces TS pre-assignment mechanism on the basis of Z-MAC [5] and resultantly solves the problem of excessive energy consumption in node idle listening. A2-MAC[6] divides time frame into two domains: signal domain and data domain, and a node respectively adopts no persistent CSMA mechanism to send control information and TDMA for data in two time domains, which improves channel usage in equity and energy saving than CSMA. VeMAC [7] assumes that each vehicle is equipped with two transceivers: one is tuned to the control channel and the other to the service channel. Its time slot reservation procedure for the control channel is very similar with ADHOC MAC. ASAS [8] introduces an Adaptive TDMA Slot Assignment Strategy (ASAS) for VANET based on clustering of vehicles. The main aim of this work is to provide a MAC layer protocol that can reduce inter-cluster interference under different traffic loading conditions without having to use expensive spectrum and complex mechanisms such as CDMA or OFDMA. CDC-MAC [9] employs a cooperative node selection algorithm which is based on weighted residual energy, and gets an improvement in energy consumption balance of entire network, but also a long delay. WTE-MAC [10] adopts a virtual tunnel (VT) mechanism, namely, a node in the link estimates wake-up time for itself by the information of its adjacent node to obtain the synchronization of sending and receiving node, which improves time delay feature of the protocol.

It can be concluded that the need of mobile multi-hop networking of WSN application is not considered in above MAC protocols. Besides, for the available protocols at present, the problem of large time delay and poor equity is widespread for dynamic TSA, and communication time interval is strictly limited to time frame length for the group and schedule in time frame, causing a poor real-time performance of burst data transmission in static TSA, while algorithm is too complicate to ensure real-time performance of burst data transmission in mixed TSA. These problems make it difficult to accommodate current TSA protocols to the application situations of ground wireless sensor network. 


\subsection{Network Model}

The network model is shown as Figure 1.

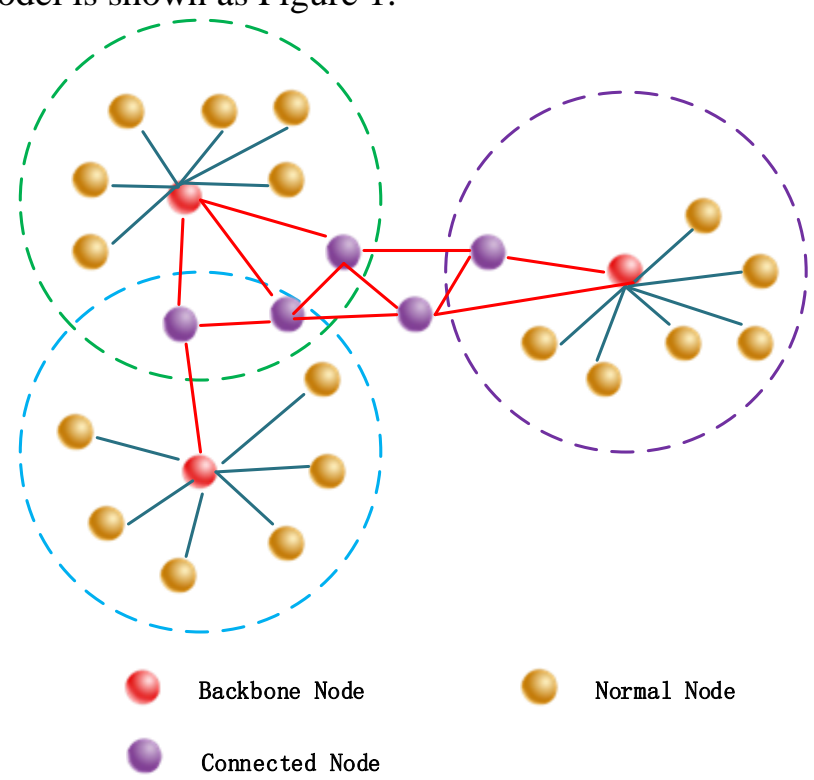

Figure 1. Network Model

It was hypothesized that:

a. The backbone nodes and connected nodes can move at low-speed, with the automated vehicle communication application. All backbone nodes and connected nodes have special power supply capability to supportin-network data aggregation and data forwarding.

b. A virtual backbone is build-up with all backbone nodes and connected nodes.

c. The normal nodes are stationary, with sufficient computational power to support different MAC protocol and data processing. The normal nodes are specified not to communicate with each other, for prolonging the normal node lifetime.

$\mathrm{d}$. The wireless link is symmetrical, having same power consumption.

e. The network capacity is 128 nodes.

\section{Protocol Design}

Protocol design mainly includes frame structure design, node storage table design, slot resource assignment scheme, slot selection\& release, conflict resolution, and mobile node design.

\subsection{Frame Structure Design}

Figure 2 pictures that a meta have been broken into two parts: control frame and data frame. The control frame is divided into Ntime-slot. $\mathrm{N}$ is the mumble of the nodes in the network, which is set to 32 in this paper. A time-slot is assigned to each node to send control packet. Each node has an opportunity to send control packet during controlling minislot. 


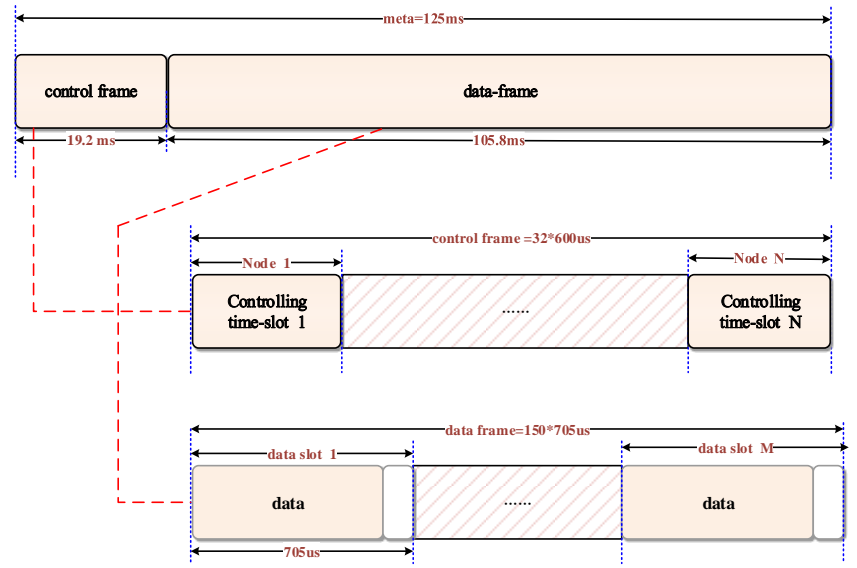

Figure 2. Frame Structure

The data sub-frame is divided into Mtime-slot. M needs to be slightly larger than network capacity, for the protocol requires that all nodes in two hops should not be in the same time-slot. Mis set to 150 in this paper. Each node would have at least one time-slot during the network load is extremely huge

\subsection{Node Storage Table Design}

The node in the network can confirm the neighbor node, after one period of information exchange. It was hypothesized that each node in the network can send and receive data, and have the neighbor node. It was hypothesized that the communication among the nodes is managed by given timeslot assignment algorithm, and the nodes are made to fit for the network topology changes. The node storage table is the key to the assignment of idle slot resource in the network and the release after using slot resource. What the node storage table represents determined if the node is able to quickly response to the network transmission.

The node storage table includes node information and slot assignment status, the specific format are shown as followed: (the maximum number of subnet nodes is 32 )

\{

a. Node number is represented in 5 bit.

b. Required time-slot number is represented in 4 bit. (Required time-slot number should be no more than half of the total number of time-slot.)

c. The releasing state of time-slot is represented in 1 bit. (1expressnon-release, 0 express release)

d. One-hop neighbor is represented in 4 bit.

e. Two-hops neighbor is represented in 4 bit.

$\mathrm{f}$. Active condition of $\mathrm{N}$ data time-slots is represented in $1 *$ Nbytes.

\}

The specific legend are shown in followed figures.

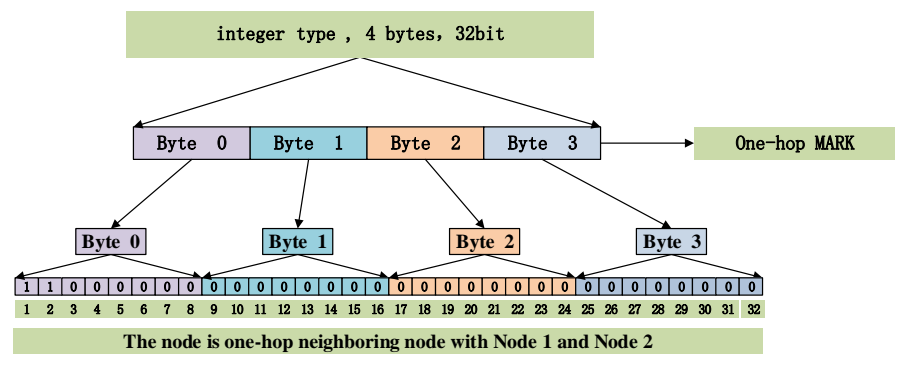

Figure 3. One-hop Neighboring Relationship Expression 


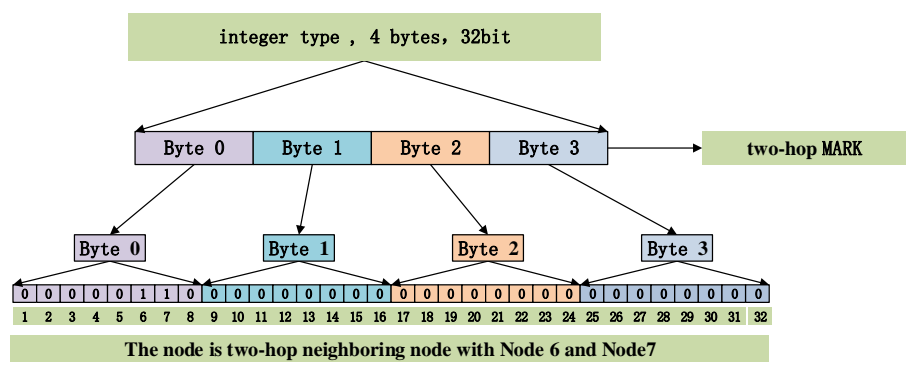

\section{Figure 4. Two-hop Neighboring Relationship Expression}

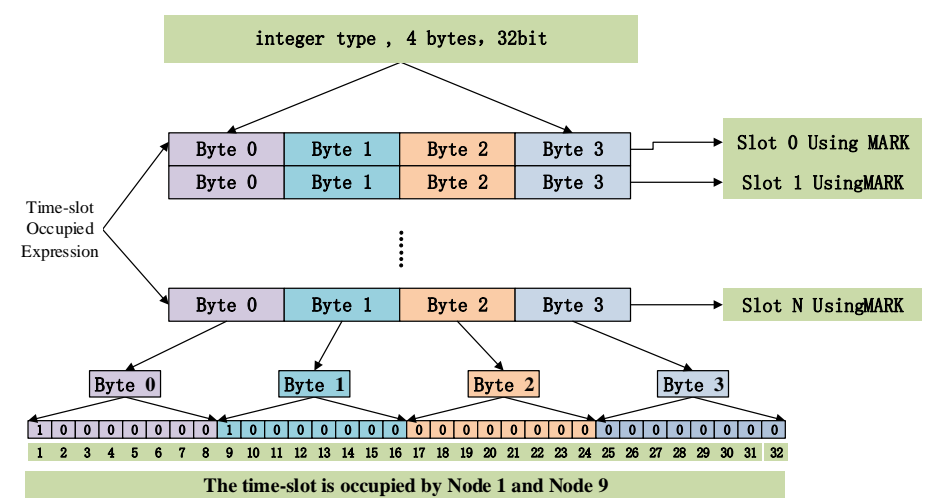

Figure 5. Time-slot Occupied Expression

Description:

'0' represents ' not' ,' 1' represents ' yes' in 8 bits.

Node location is coordinates in space of the node, for example, $(105.52,109.43)$.

The release slot mark is ' 1 ' if never released, and is ' 0 ' if released.

In order to maintain fairness, one backbone node or connected node has the time-slots must not exceed half of the maximum number of neighborhood nodes, and normal node has one time-slot at most.

The time-slot requirement number of neighborhood nodes and the release slot mark is default value during initialization, which is ' 0 '.

The slot table update process is shown in Figure 6.

Description:

Slot table update process contains two conditions, specific analyses are shown as followed.

If the slot status in local slot table is same with the corresponding slot status, the slot status remain unchanged.

If the slot status in local slot table is different with the corresponding slot status, there would be two conditions.

a. If the node number is same with the time-slot number, it' $\mathrm{s}$ needed to judge whether the node is one-hop neighboring node with local node. (1) If the node is one-hop neighboring node, the slot status remain unchanged. (2) If the node is not, the slot status would update with the latest status. For example, the time-slot 2' s status of Node 1 and Node 2 is ' 1 ' , while the time-slot 2' $\mathrm{s}$ status received is ' 0 '. It would judge whether the node 1 is one-hop neighboring node with node 2 . If the relationship is onehop neighboring, the time-slot 2' s status remain ' 1 ' . If the relationship is not one-hop neighboring, the time-slot 2' s status would update to ' 0 ' .

b. If the node number is different with the time-slot number, it' $s$ needed to judge whether the slot status of local node is ' 1 ' . (1) If the slot status is not ' 1 ' , the slot 
status would update with the latest status. (2) If the node status is ' 1' , it' s needed to judge whether the node occupying the slot is two-hop neighboring node with local node. If the node is two-hop neighboring node, the slot status would update with the latest status. If the node is not, it would estimate the control packet types. When the control packet type is ' 1' , the slot status would update with the latest status. When the control packet type is not ' 1 ', the slot status remain unchanged.

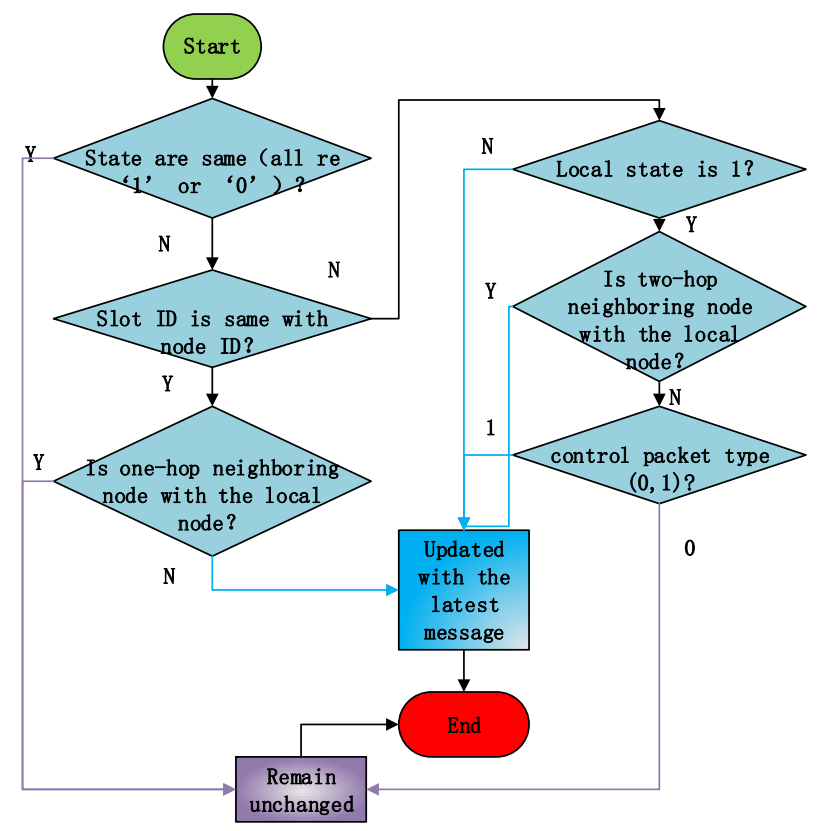

Figure 6. Slot Table Update Process

\subsection{Slot Resource Assignment Scheme}

\subsubsection{Slot Assignment Model}

The ratio of control frame and data frame is $1: 1$. If the cyclic numbers is not less than 7 , it is not allowed to request time-slot. It would be dynamic reconfigured after 8 cycles, the concrete model is shown in Figure 7.

According to the maximum requirements of nodes, fixed slot assignment protocol assigns needed time-slots to corresponding nodes, each node has at least one time-slot.

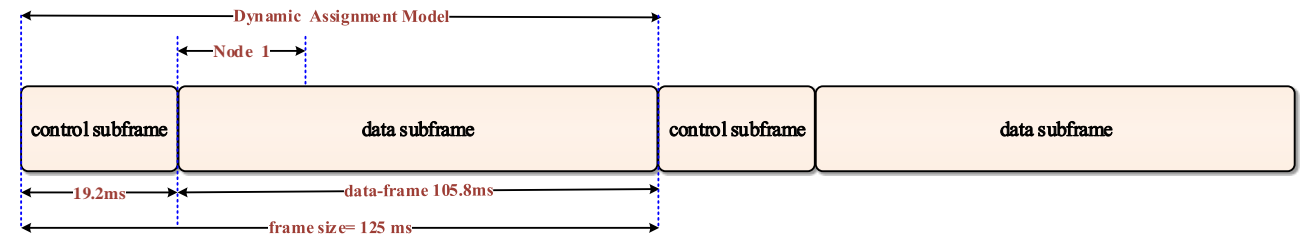

Figure 7. Dynamic Time Slot Assignment Model

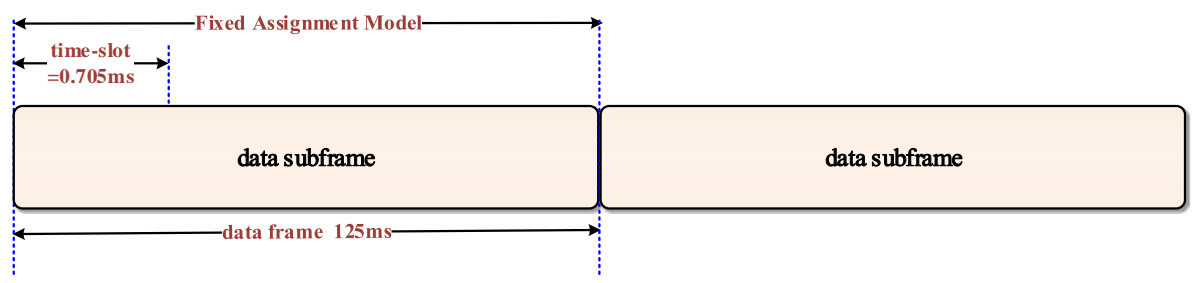

Figure 8. Fixed Time Slot Assignment Model 


\subsubsection{Virtual Backbone Choice}

The connected dominating set (CDS) is an effective method to build virtual backbone subnet. It is familiar with cluster structure, nodes in dominating set are playing an important role in information dissemination and relay. Which means information can exchange in CDS. CDS has strengths in dynamic topology control. Building virtual backbone network while topology changing makes packet trunking finished in CDS. It would reduce energy consumption, extend network lifetime.

A CDSgraph $G$ is composed by a set of connected nodes. Each node in graph $G$ is member of CDS, or one-hop neighboring node of the member. CDS defined a set of connected nodes, which need not to connect with a concentrated dominating node. Choosing dominating set is determined by neighboring degree and remaining battery life. The nodes in CDS are virtual backbone nodes, which are the only nodes can select and release time-slot dynamically.

\subsubsection{Slot Assignment Process}

The virtual backbone nodes send control packet, the time-slot are choosing based on timeslot assignment algorithm. There are three phase - reservation phase, dynamic assignment phase and contention resolution phase. Reservation phase assign one time-slot to each node after receiving neighbor information, the number of the node corresponds to the number of the time-slot, and the local time-slot information are updated. Dynamic assignment phase is mainly about the reuse of time-slot and the use of free time-slot. Contention resolution phase arbitrates the conflict happened during time-slot assignment process, and notifies in the next frame. Figure 9 pictures the detailed flow chart.

The distribution restriction makes each node getting time-slot if needed, node in twohop neighboring not having the same time-slot. If there is no slot request from a node, the preempted time-slot would be released, and is informed to other neighboring nodes. The time-slot released can be used by other nodes in this cycle or the next cycle.

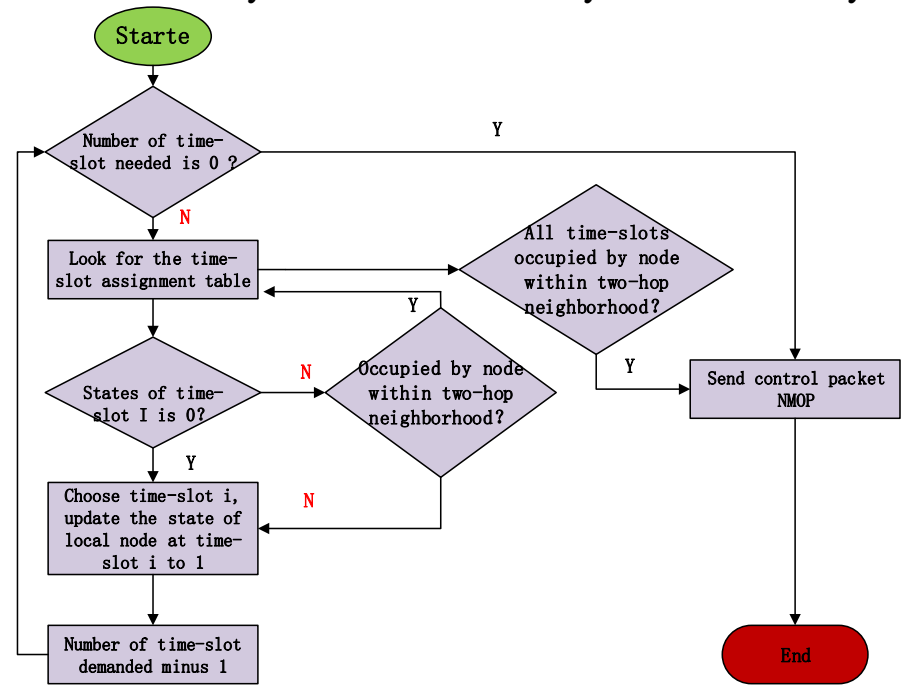

Figure 9. Slot Assignment Flow Diagram

Dynamic time-slot assignment process is analyzed as followed:

a. Without losing control packet, there would not be assignment conflict in the algorithm.

b. The node havingtime-slot requirements would get at least one usable time-slot, by interaction of control packet among neighboring nodes.

c. The nodes at a distance of more the two-hop neighborhood, can multiplex the time-slot. Because the nodes are informed with the time-slot assignment state in two-hop 
neighborhood, there is no interaction of time-slot assignment state among the nodes at a distance of more the two-hop neighborhood.

\subsection{Slot Selection}

\subsubsection{Slot Selection Release}

When one new node joins the network, it' s needed to sniffer at least two cycles to get the neighboring nodes and the slot assignment information, and choose the free time-slot or reusable time-slot. If there is no free time-slot, it should be waiting until the next cycle to assign. When more new node join the network, it should request for time-slot according to the node ID size of order.

When the node needs to occupy data time-slot, it' s necessary to choose free time-slot and try to meet the demand of local nodes after looking up local saved time-slot assignmentTable, and send message to the corresponding control packet.

When the number of time-slot requested is smaller than the ones occupied, it would release more free time-slots and send to the corresponding control packet.

\subsubsection{Slot Status Diagram}

a. If a time-slot has not been occupied, the time-slot status is ' free' .

b. If a time-slot is assigned to a node from free time-slots, the time-slot status is " predistribution'.

c. If there is conflict, the time-slot status returns to ' free' .

d. If there is no conflict, the time-slot status goes to ' seizure' .

e. After the use of occupied time-slot, as cycletimeout values $\mathrm{C}=0$, the time-slot status returns to ' free'.

The cycletimeout valuesC is specified by the system. The user cannot occupy the timeslot all the time after assigned to, and must abort the occupying after certain time-frames. Cindicates the frame number of the currently used time-slot which continues being using. $\mathrm{C}$ would minus one after one frame.

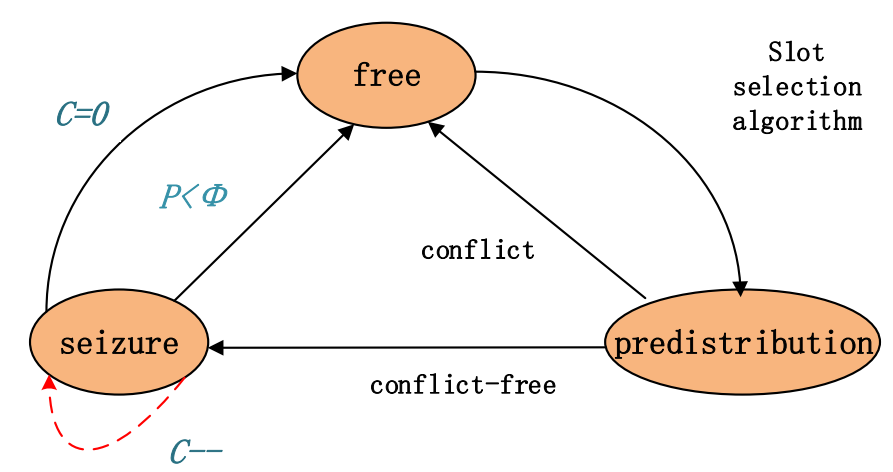

Figure 10. Slot Status Diagram

As shown in Figure 14, Prepresents priority level in the business flow. Considering there would be a heavy load, the free time-slots in one frame may not meet the needs of the new business. To provide quality of service $(\mathrm{QoS})$ guarantee to high priority applications, two prioritythresholds () are set. The business flow having priority higher than can preempt the occupied time-slot from the business flow having priority lower than. In this way, the business flow having priority higher than can consider the occupied time-slot of the business flow having priority lower than as a free time-slot, which can be reuse. 


\subsection{Conflict Resolution}

In the time-slot assignment process, if there were nodes in two-hop neighborhood occupying one time-slot, conflicts are tackled as followed.

a. When receiving node gets the time-slot assignment information from the sending node, it' $s$ needed to judged weather there is a conflict between the time-slot occupied by the sending node and the time-slot occupied by the other node in local time-slot assignment table.

b. If there is a conflict between the time-slot occupied by the sending node and other node, it should judge weather the two nodes in conflict are one-hop neighboring with local node.

c. If the two nodes in conflict are one-hop neighboring node, the node with a smaller ID occupies the time-slot, and the time-slot of the other node update to 0.If the two nodes in conflict aren' t one-hop neighboring node, time-slot assignment table would be updating based on the slot assignmentprocess in Section 2.c.3.

\section{Theoretical Performance Analysis}

The throughput and the access delay of the dynamic time-slot assignment protocol is the oryanalyze in this section. It was hypothesized that there is no loss of the data packets in the network during sending and receiving, and sending packets are as same as receiving packets. THROUGHPUT is defined as the summation of the sending data packets in the network in a unit time. ACCESS DELAY is defined astime difference between packet generation and sending packet of each node.

From the above definition, the equation of THROUGHPUT can be expressed as:

$$
\mathrm{P}=
$$

The equation of AVERAGE ACCESS DELAY can be expressed as:

$$
\mathrm{TD}=
$$

Where $P$ denotes throughput, $L_{i}$ is the sending packet length in one frame of each node $i, T_{f}$ is frame length, $N$ is the number of nodes in the network, $T D$ is the average delay, $s_{i}$ is the number of time-slots occupied by node $i$, and $s r$ is the number of time-slots needed by each node.

Assuming there is $\mathrm{N}$ nodes in the network, the number of two-hop neighboring nodes of node $i$ is $M_{i}$, each time-a lot would send a packet which having a length of $L_{0}$ bits, the length of control sub-frame in time-frame is $T c t r l$, the length of each time-slot in data sub-frame is $T_{0}$, and the length of time-frame can be given as:

$$
\mathrm{Tf}=\mathrm{Tctrl}+\mathrm{Mmax} * \mathrm{~T} 0
$$

When the network is in a full load condition, each node would need at least one time-slot to send packet. Each node could get one time-slot based on the dynamic time-slot assignment protocol, and the length of packet sent by each node is $L_{0}$ bit. The network throughput can be expressed as:

$$
\mathrm{P}==
$$

Assumingthe number of time-slots occupied by each node is 1, ACCESS DELAY can be given as:

$$
\mathrm{TD}==\mathrm{Tctrl}+\mathrm{Mmax} * \mathrm{~T} 0
$$

Assumingthe network includes at least 4 nodes, the value range of $M_{\max }$ is Mmax , and the value range of THROUGHPUT could be:

The value range ofAVERAGE ACCESS DELAY could be:

$$
\text { Tctrl+ T0* } 3 \text { TDTctrl+N* T0 }
$$

From the aboveequations, it can be inferred that:

a. The throughput generated by the protocol is related to the frame size. With $\mathrm{N}$ (the number of nodes in the network) increasing, the throughput generated by the protocol increases linearly. For the time-slot can be reused, the overall throughput is much larger than the one statically assigned. 
b. The minimum access delay is a fixed value, which wouldn' $t$ increase with the number of nodes increasing. But the maximum access delay increases with the number of nodes increasing. The protocol is with a fully dynamic assignment, which choose slot by the exchange of control packet. Once there is a loss of the control packet, there may be an assignment conflict, which couldn' t be avoided within a short time. This could result in the access delay increasing.

\section{Result Analysis}

This paper presents a simulation of the dynamic timeslot assignment protocolbased on OPNET. The simulation is mainly about the distribution and possession of the time-slot resource, statistics include time delay, time-slot occupancy, and throughput. The simulation is to verify the feasibility and to analyze the performance of the protocol. Figure 11 pictures demand framework of the dynamic slot assignment protocol.

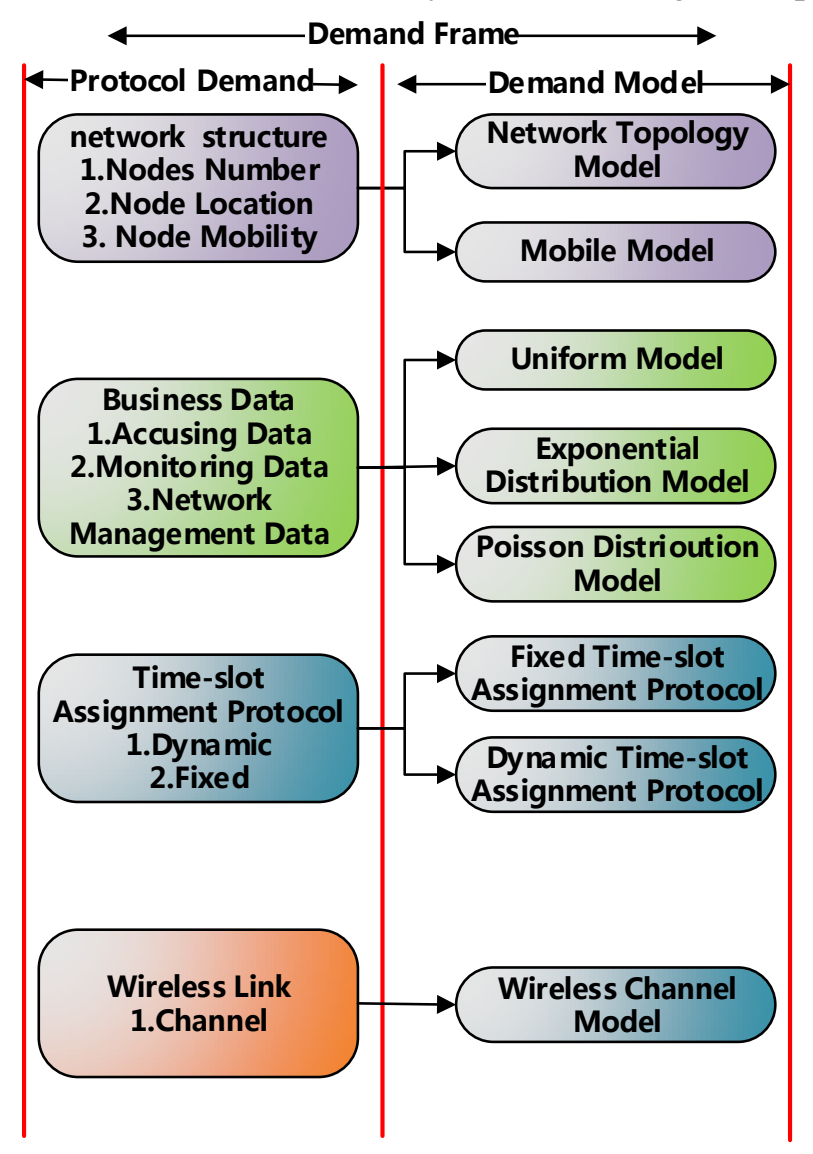

Figure 11. Demand Frame Work for Protocol

\subsection{Simulation Design}

Figure 12 and Figure 13 shows the basic configuration parameters and topology of dynamic timeslot assignment protocolbased on OPNET. 


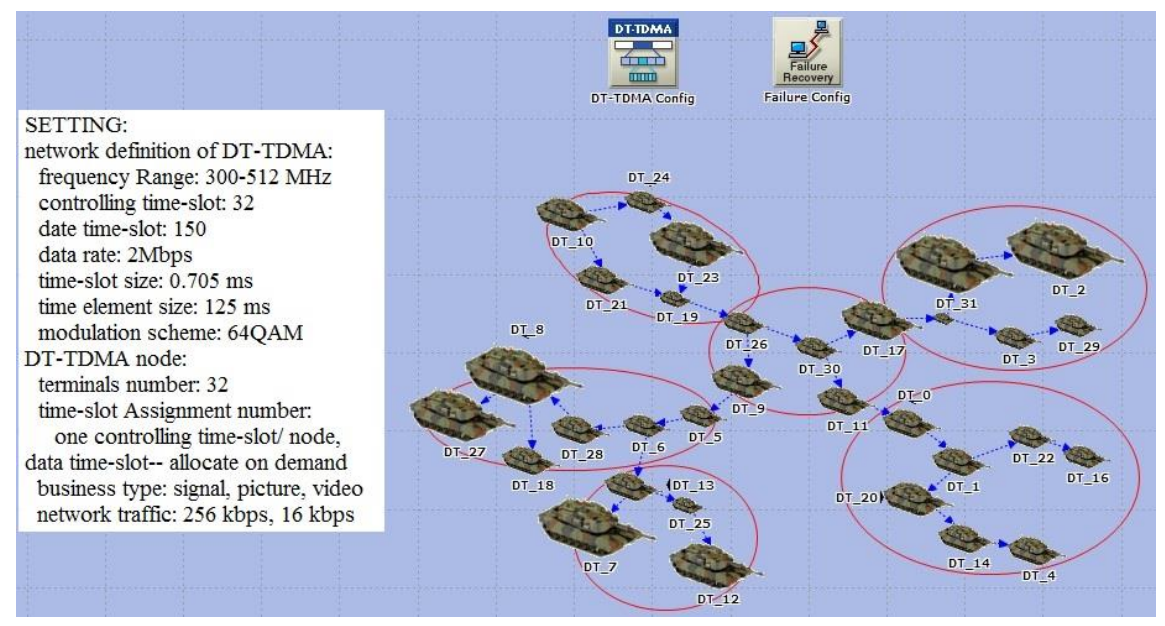

Figure 12. Simulation Scene

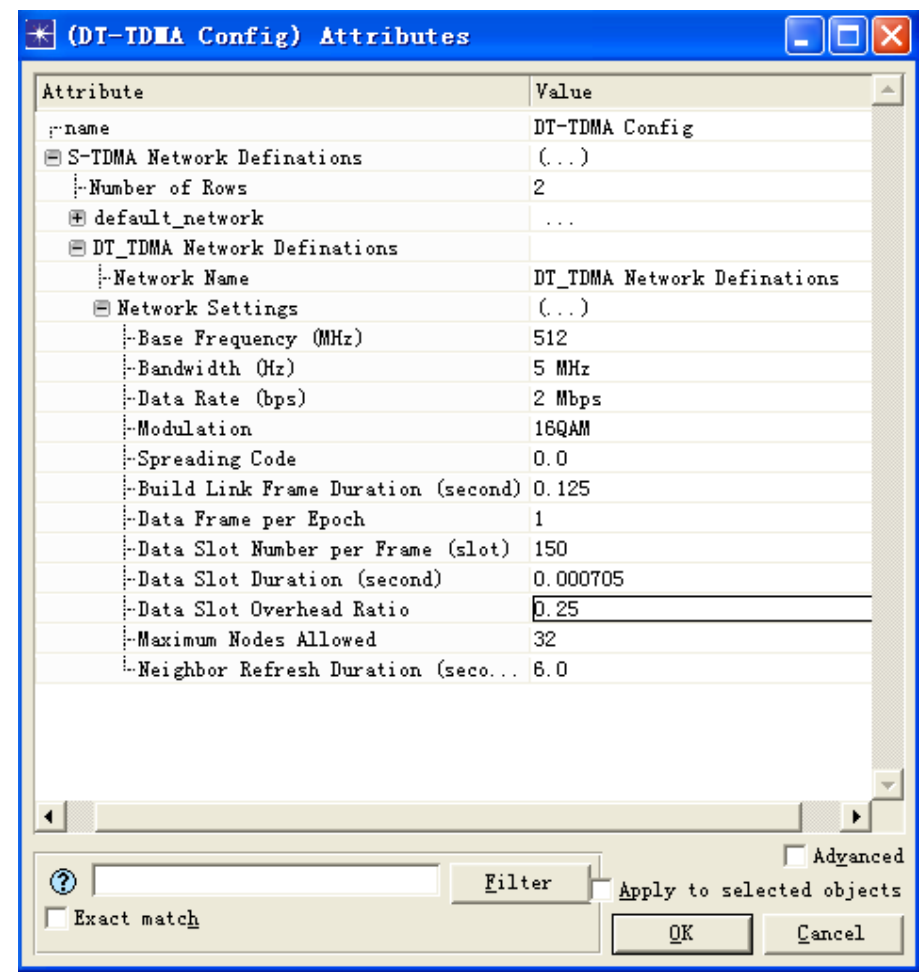

Figure 13. Main configuration of simulation

\subsection{Performance Analysis}

\subsubsection{Analysis of Access Delay}

a) Analysis of Access Delay under Different Network Load

Parameter setting: The user rate is $2 \mathrm{Mbps}$, the number of nodes is 32, maximum number of allowable hops is 4 , and maximum number of two-hop neighboring nodes is 32. Each node has a same load, and the size of the packet generated is 128 bytes. Data packet is generated with the time obeyed uniform distribution, which are $0.008 \mathrm{~s}, 0.09 \mathrm{~s}$, $0.1 \mathrm{~s}$, and $0.11 \mathrm{~s}$ respectively. The specific result is shown in Table 1 .

The access delay of the dynamic timeslot assignment protocol is smaller than the fixed protocol, the difference of the access delay increased with the load in network increasing. 
This is because the dynamic timeslot assignment is a completely dynamic assignment protocol, which makes the nodes having nodes requirement to occupy the nearest usable time-slot. The fixed timeslot assignment is a fixed protocol, which makes each node to occupy the time-slot assigned to, it' $\mathrm{s}$ obvious that the access delay is larger than the dynamic protocol.

With the load increasing, the access delay would slightly increase. This is because the dynamic timeslot assignment would assign every usable time-slot to the node, the reuse density of time-slot is high. The fixed timeslot assignment makes each node to occupy the time-slot assigned to. There would be time-slot beyond two-hop not being assigned to any node, the reuse density of time-slot is low, and the access delay increases correspondingly.

Table 1. Analysis of Access Delay under different Network Load

\begin{tabular}{|c|c|c|c|c|c|}
\hline \multicolumn{2}{|c|}{} & \multicolumn{4}{|c|}{ Total network load (Kbps) } \\
\cline { 3 - 6 } & & 147 & 164 & 183 & 205 \\
\hline \multirow{2}{*}{$\begin{array}{c}\text { Average access } \\
\text { delay (s) }\end{array}$} & Fixed & 0.338 & 0.5 & 2.34 & 4.12 \\
\cline { 2 - 6 } & Dynamic & 0.295 & 0.25 & 0.4 & 0.38 \\
\hline
\end{tabular}

Table 2. Analysis of Access Delay under different Network Size

\begin{tabular}{|c|c|c|c|c|c|}
\hline \multicolumn{2}{|c|}{} & \multicolumn{5}{|c|}{ Node Number } \\
\cline { 3 - 6 } & & 8 & 16 & 24 & 32 \\
\hline \multirow{2}{*}{ Average access delay(s) } & Fixed & 0.128 & 0.448 & 1.78 & 2.87 \\
\cline { 3 - 6 } & Dynamic & 0.08 & 0.213 & 0.518 & 0.888 \\
\hline
\end{tabular}

Table 3. Analysis of Access Delay under different Business Model

\begin{tabular}{|c|c|c|c|}
\hline \multicolumn{2}{|c|}{} & \multicolumn{2}{|c|}{ Business Model } \\
\cline { 3 - 4 } & Business Model No.1 & Business Model No.2 \\
\hline \multirow{2}{*}{$\begin{array}{c}\text { Average access } \\
\text { delay(s) }\end{array}$} & Fixed & 0.448 & 5.179 \\
\cline { 2 - 4 } & Dynamic & 0.213 & 0.9 \\
\hline
\end{tabular}

b) Analysis of Access Delay under Different Network Size

Parameter setting: The user rate is $2 \mathrm{Mbps}$, and the maximum number of allowable hops is 4 . The number of nodes are $8,16,24$, and 32respectively.The maximum number of two-hop neighboring nodes are 8, 16, 24, and 32correspondingly. Each node has a same load, and the size of the packet generated is 128 bytes. Data packet is generated with the time obeyed uniform distribution, which is $0.1 \mathrm{~s}$. The specific result is shown in Table 2.

From the table we notice that there was a tiny difference between the access delays of the two protocol, and the access delay of the dynamic protocol is smaller than the fixed protocol. With the load in network increasing, the access delays of the two protocol increase, but increasing amplitude of the dynamic protocol is smaller than the fixed protocol. This is because with major nodes, the time-slots of the fixed protocol are not reused, the major load in the network makes the access delay increase. While the timeslots of the dynamic protocol are reused, the control slot becomes large, but the increasing amplitude of the subsequent access delay is smaller than the fixed protocol.

c) Analysis of Access Delay under DifferentBusiness Model

Parameter setting: The user rate is $2 \mathrm{Mbps}$, the number of nodes is 32, maximum number of allowable hops is 4 , and maximum number of two-hop neighboring nodes is 32. The data generated by business model didn't exceed the channel capacity. 
Business model 1: Each node has a same load, and the size of the packet generated is 128 bytes. Data packet is generated with the time obeyed uniform distribution, which is $0.1 \mathrm{~s}$.

Business model 2: Each node has a different load, and the size of the packet generated is 128 bytes. Data packet is generated at different time intervals, which are 0 (Node 0 , Node 6, Node 10, Node 14, and Node 15), 0.04s (Node 1, Node 2, Node 3, and Node 4), 0.06s (Node 5, and Node 7), 0.08s (Node 8, and Node 9), 0.1s (Node 11, Node 12, and Node 13), and $0.5 \mathrm{~s}$ (the rest nodes).The operation time is 1 minute, and the specific result is shown in Table 3.

From the table we notice that there was a large difference between the access delays of the two protocols under business model No.2, and the difference was smaller under business model No.1. The dynamic time-slot assignment protocol shows a smaller access delay performance. This is because the two protocols having different time-slot assignment methods, and the dynamicprotocol makes the nodes having nodes requirement to occupy the nearest usable time-slot.

\subsubsection{Throughput Analysis}

a) Analysis of Throughputs under Different Network Load

Parameter setting: The user rate is $2 \mathrm{Mbps}$, the number of nodes is 32, maximum number of allowable hops is 4, and maximum number of two-hop neighboring nodes is 32. Each node has a same load, and the size of the packet generated is 128 bytes. Data packet is generated with the time obeyed uniform distribution, which are $0.05 \mathrm{~s}, 0.06 \mathrm{~s}$, $0.07 \mathrm{~s}, 0.08 \mathrm{~s}$, and $0.09 \mathrm{~s}$ respectively. The operation time is 1 minute, and the specific result is shown in Table 4.

From the above table it can be seen that there was a tiny difference between the throughputs of the two protocol, the throughput of the dynamicprotocol is larger than the fixed protocol with the load increasing. The time-slot resource is greater than the network demand when the load is low, both protocols make the assignment satisfies the needs of each node. With the load increasing and being close to the channel rate, the fixed protocol makes the node only occupies the time-slot assigned to, The number of time-slots in each frame on the dynamicprotocol is the number of two-hop neighboring nodes, which is less than or equal to the number of the nodes in network. So the reuse rate of time-slot is high, the throughput of the dynamicprotocol is larger than the fixed protocol. When the load exceeds the channel rate, the throughput of the dynamicprotocol drops gradually. Because the data would cumulate to the next frame if failed to fully process. Even though the timeslot can be reused, the overall throughput drops once the arrival rate exceeded the service rate

b) Analysis of Throughputs under Different Network Size

Parameter setting: The user rate is $2 \mathrm{Mbps}$, and the maximum number of allowable hops is 5 . The number of nodes are $8,16,24$, and 32 respectively. The maximum number of two-hop neighboring nodes are 8, 16, 24, and 32 correspondingly. Each node has a same load, and the size of the packet generated is 128 bytes. Data packet is generated at 0.1-second intervals. The specific result is shown in Table 5.

From the above table it can be seen that there was a tiny difference between the throughputs of the two protocol when the node number is small, the throughput of the dynamicprotocol is larger than the fixed protocol when the node number is larger. The frame size of the two protocols are approximative with few nodes, and both protocols can satisfy the needs of the load when the total load is small. As the nodes increase, the largest hop number in the network is 5, the number of time-slots in each frame on the dynamicprotocol is the number of two-hop neighboring nodes, which is less than the number of the nodes in network. The number of time-slots in each frame on the fixed protocol is the number of the nodes in network. Which makes the reuse rate of the 
dynamicprotocol is larger than the fixed protocol, and the throughput of the dynamicprotocol increases as well.

c) Analysis of Throughputs under DifferentBusiness Model

Parameter setting: The user rate is $2 \mathrm{Mbps}$, the number of nodes is 32, maximum number of allowable hops is 4 , and maximum number of two-hop neighboring nodes is 32. The data generated by business model didn't exceed the channel capacity.

Business model 1: Each node has a same load, and the size of the packet generated is 128 bytes. Data packet is generated with the time obeyed uniform distribution, which is $0.1 \mathrm{~s}$.

Business model 2: Each node has a different load, and the size of the packet generated is 128 bytes. Data packet is generated at different time intervals, which are 0 (Node 0 , Node 6, Node 10, Node 14, and Node 15), 0.04s (Node 1, Node 2, Node 3, and Node 4), 0.06s (Node 5, and Node 7), 0.08s (Node 8, and Node 9), 0.1s (Node 11, Node 12, and Node 13 ), and $0.5 \mathrm{~s}$ (the rest nodes).The operation time is 1 minute, and the specific result is shown in Table 6 .

From the above table it can be seen that there was a large difference between the throughputs of the two protocols under business model No.2, the throughput of the dynamicprotocol is about $50 \%$ higher than the fixed protocol, and the difference was smaller under business model No.1. This is because the packet sending of business model No.2 is more dynamic, the dynamicprotocol can make use of the smaller frame size and be more dynamic to adapt to the dynamic change of the business.

Table 4. Analysis of Throughputs under different Network Load

\begin{tabular}{|c|c|c|c|c|c|c|}
\hline \multicolumn{2}{|c|}{} & \multicolumn{5}{|c|}{ Total network load(Kbps) } \\
\cline { 3 - 7 } & 183 & 205 & 235 & 275 & 327 \\
\hline \multirow{2}{*}{$\begin{array}{c}\text { Average } \\
\text { Throughputs(Kbps) }\end{array}$} & Fixed & 164 & 164 & 164 & 164 & 164 \\
\cline { 3 - 7 } & Dynamic & 179 & 202 & 226 & 221 & 217 \\
\hline
\end{tabular}

Table 5. Analysis of Throughputs under different Network Size

\begin{tabular}{|c|c|c|c|c|c|}
\hline \multicolumn{2}{|c|}{} & \multicolumn{4}{|c|}{ Node Number } \\
\cline { 3 - 6 } & 8 & 16 & 24 & 32 \\
\hline \multirow{2}{*}{$\begin{array}{c}\text { Average } \\
\text { Throughputs(Kbps) }\end{array}$} & Fixed & 164 & 164 & 164 & 164 \\
\cline { 2 - 6 } & Dynamic & 144 & 217 & 324 & 447 \\
\hline
\end{tabular}

Table 6. Analysis of Throughputs under different Business Model

\begin{tabular}{|c|c|c|c|}
\hline & \multicolumn{2}{|c|}{ Business Model } \\
\hline & & Business ModelNo. 1 & Business ModelNo. 2 \\
\hline \multirow{2}{*}{$\begin{array}{c}\text { Average } \\
\text { Throughputs(Kbps) }\end{array}$} & Fixed & 162 & 102 \\
\hline & Dynamic & 163 & 164 \\
\hline
\end{tabular}

\subsubsection{Timeslot Occupancy Analysis}

a) Demand Analysis

Parameter setting: The user rate is $2 \mathrm{Mbps}$, the number of nodes is 8 , maximum number of allowable hops is 4 , and maximum number of two-hop neighboring nodes is 8 . The size of the packet generated is 128 bytes. Data packet is generated at different time intervals, which are 0.05s (Node 0), 0.06s (Node 1), 0.07s (Node 4, and Node 5), and $0.08 \mathrm{~s}$ (Node 7). And there is no data generated by Node 2, Node 3, and Node 6 . The operation time is 1 minute, and the specific time-slot assignment proportion is shown in Table 6.

A quick look at the table above indicates that the dynamic assignment protocol achieves the function of dynamic assignment on demand, the fixed assignment protocol only makes use of preplanned assigned time-slots. The dynamic protocol dynamically 
uses the nearest usable time-slot based on the assignment information of the nodes within two-hop neighbor, and the time-slot can be occupied if it is free and has no conflict, which makes the resource utilization high. The fixed protocol makes the node only occupies the time-slot assigned to, the other free time-slots cannot be occupied, which leads to the waste of time-slot resource.

Table 7. Time-slot Assignment Proportion under different Node Load

\begin{tabular}{|c|c|c|c|c|c|c|c|c|c|}
\hline \multirow{2}{*}{} & \multicolumn{7}{|c|}{ Node Number } \\
\cline { 2 - 10 } & 0 & 1 & 2 & 3 & 4 & 5 & 6 & 7 \\
\hline \multicolumn{2}{|c|}{ Timeslot demand } & 4 & 4 & 0 & 0 & 3 & 3 & 0 & 3 \\
\hline $\begin{array}{c}\text { Assignment } \\
\text { Proportion (\%) }\end{array}$ & Fixed & 12.5 & 12.5 & 12.5 & 12.5 & 12.5 & 12.5 & 12.5 & 12.5 \\
\cline { 2 - 10 } & Dynamic & 50 & 12.5 & 0 & 0 & 25 & 12.5 & 0 & 37.5 \\
\hline
\end{tabular}

Table 8. Time-slot Assignment Proportion under same Node Load

\begin{tabular}{|c|c|c|c|c|c|c|c|c|c|}
\hline & \multicolumn{7}{|c|}{ Node Number } \\
\cline { 2 - 10 } & 0 & 1 & 2 & 3 & 4 & 5 & 6 & 7 \\
\hline \multicolumn{2}{|c|}{ Timeslot demand } & 2 & 2 & 2 & 2 & 2 & 2 & 2 & 2 \\
\hline $\begin{array}{c}\text { Assignment } \\
\text { Proportion (\%) }\end{array}$ & Fixed & $\begin{array}{c}12 . \\
5\end{array}$ & 12.5 & 12.5 & 12.5 & 12.5 & 12.5 & 12.5 & 12.5 \\
\cline { 2 - 10 } & Dynamic & 25 & 12.5 & 12.5 & 12.5 & 12.5 & 12.5 & 12.5 & 25 \\
\hline
\end{tabular}

b) Fairness Analysis

Parameter setting: The user rate is $2 \mathrm{Mbps}$, the number of nodes is 8 , maximum number of allowable hops is 4 , and maximum number of two-hop neighboring nodes is 8 . Each node has a same load, and the size of the packet generated is 128 bytes. Data packet is generated at 0.1-second intervals. The specific time-slot assignment proportion is shown in Table 8.

A quick look at the table above indicates that the number of time-slots assigned to each node using the two protocols have not much difference, the assignment is effective while ensuring certain fairness, the nodes having time-slot requirement have at least one timeslot assigned to. The dynamic protocol assigned more time-slots, and makes full use of the time-slot resource. The fixes protocol has a smaller difference range of the time-slot number of each node, which shows greater fairness.

\subsubsection{Discussion}

Dynamic TSA protocol has a higher channel utilization ratio than a fixed one, and the lager number of nodes and load, the higher utilization ratio.

Dynamic TSA protocol has a lager network throughput in condition of not exceeding channel capacity than a fixed one, and the more nodes, the greater the difference of throughput.

Dynamic TSA protocol has a shorter average access delay than a fixed one, and the lager number of nodes and load, the greater the difference of delay.

It suggests that dynamic TSA protocol is more applicable to a large scale and load of ground wireless network, because it achieves the efficient dynamic assignment of resources, and further ensures the node equity in distributed ground wireless network. 


\section{Conclusion and Future Work}

For the need of short delay in mobile multi-hop, equity and burst traffic application in ground wireless network, a TDMA-based dynamic TSA protocol is proposed. It employs TS reuse strategy and virtual backbone mechanism beyond two hops, which achieves flexible assignment of TS resource, reduces end-to-end delay, prolongs the network life cycle, improves real-time transmission performance in periodic data sensory and burst data, promotes channel access equity of sensor node, and increases network throughput.

According to the features of protocol, control packet structure or content can be improved to reduce network overhead, besides QoS is added to form a differentiated priority for different types of transactions and different levels of nodes and finally improve the network service quality.

\section{Acknowledgement}

This paper is supported by the Natural Science Foundation of Hubei Province (No. 2013CFC009), the International Science \& Technology Cooperation Program of China under grant No. 2013DFA12460, and the department of Education of Hubei Province (B2013032).

\section{References}

[1] V. Rajendran, K. Obraczka and J. J. Garcia-Luna-Aceves, "Energyefficient, collision-free medium access control for wireless sensor networks", Proceedings of the 1st International Conference on Embedded Networked Sensor Systems (SenSys '03), Los Angeles, Calif, USA, (2003), pp. 181-192.

[2] L. F. W. Van Hoesel and P. J. M. Havinga, "A lightweight medium access protocol for wireless sensor networks", 1st International Workshop on Networked Sensing Systems (INSS), Tokyo, pp. 205-208.

[3] A. Rowe, R. Mangharam and R. Rajkumar, "RT-Link: A Time-Synchronized Link Protocol for EnergyConstrained Multi-hop Wireless Networks", Proceedings of the 3rd Annual IEEE Communications Society on Sensor and Ad hoc Communications and Networks, Pittsburgh, Pa, USA, September, vol. 2, (2006), pp. 402-411.

[4] J. Guo, Y. Huo, C. Hu, T. Liang, Y. Liu and L. Zhang, "An adaptive and reliable MAC mechanism for IEEE 1609.4 and 802.11 p VANETs", Wireless Personal Multimedia Communications (WPMC), 2012 15th International Symposium, (2012), pp. 55-59.

[5] I. Rhee, A. Warrier, M. Aia and J. Min, "Z-MAC: a hybrid MAC for wireless sensor networks", IEEE/ACM Transactions on Networking, vol. 16, no. 3, (2005), pp. 511-524.

[6] S. Zhou, R. Liu, D. Everitt and J. Zic, "A2-MAC: An application adaptive medium access control protocol for data collections in wireless sensor networks", Proceedings of the IEEE Symposium on Communications and Information Technologies (ISCIT2007), Sydney, NSW, (2007), pp. 1131-1136.

[7] H. A. Omar, W. Zhuang and L. Li, "VeMAC: A TDMA-based MAC protocol for reliable broadcast in VANETs", Mobile Computing, IEEE Transactions, vol. 12, (2013), pp. 1724-1736.

[8] M. Hadded, R. Zagrouba, A. Laouiti, P. Muhlethaler and L. A. Saïdane, "An Adaptive TDMA Slot Assignment Strategy in Vehicular Ad Hoc Networks", Journal of Machine to Machine Communications, vol. 1, (2014), pp. 175-194.

[9] H. Jiaot, M. A. Ingram and F. Y. LI, "A cooperative lifetime extension MAC protocol in duty cycle enabled wireless sensor networks", Proceedings of the International Conference on Military Communications (MILCOM 2011), Baltimore, MD, (2011), pp. 896-901.

[10] J. Lee, K. Hur and D. Eom, "WTE-MAC: wakeup time estimation MAC for improving end-to-end delay performance in WSN", Proceedings of the International Conference on Military Communications (MILCOM2011), Baltimore, MD, (2011), pp. 902-907.

\section{Authors}

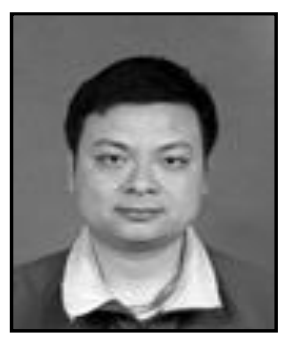

Gao Ren, he was born in Wuhan, Hubei, China, in 1979. He received the $\mathrm{Ph} . \mathrm{D}$. degree in electronic information engineering from Wuhan University, Wuhan, China, in 2012. Currently, he is an associate professor of Hubei University of Economics, Wuhan, Hubei, China. His major research interest is wireless network. 

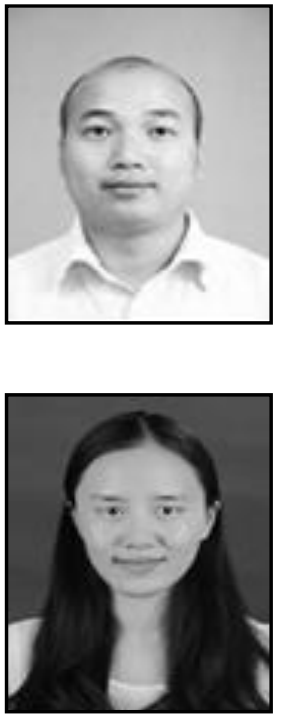

Tang Long, he was born in Hechi, Guangxi, China, in 1982. He received the $\mathrm{Ph} . \mathrm{D}$. degree in electronic information engineering from Wuhan University, Wuhan, China, in 2012. Currently, he is a lecturer of Hubei University of Economics, Wuhan, Hubei, China. His major research interest is wireless network.

Hu Wen, he was born in Yichang, Hubei, China, in 1987. She received the $\mathrm{Ph} . \mathrm{D}$. degree in electrical engineering from Wuhan University, Wuhan, China, in 2014. Currently, she is a lecturer of Hubei University of Economics, Wuhan, Hubei, China. Her research interests include Internet of Things, and modern signal-processing applications in power systems. 
International Journal of Future Generation Communication and Networking Vol. 9, No.7, (2016) 